\title{
ESTILOS PARENTALES PERCIBIDOS, PSICOPATOLOGÍA Y PERSONALIDAD EN LA ADOLESCENCIA
}

\author{
BORJA IGLESIAS ${ }^{1}$ Y ESTRELLA ROMERO ${ }^{2}$ \\ ${ }^{1}$ Unidad de Salud Mental de Vecindario, Las Palmas de Gran Canaria \\ ${ }^{2}$ Facultad de Psicología. Universidad de Santiago de Compostela
}

\begin{abstract}
Resumen: Esta investigación se plantea con el objetivo de analizar la relación entre los estilos educativos percibidos y la psicopatología y la personalidad, tal y como se definen desde el modelo de Millon, en una muestra clínica de adolescentes. Participaron en el estudio 123 pacientes ambulatorios, de edades comprendidas entre los 12 y los 19 años. Para obtener los datos de esta investigación se ha utilizado, por una parte, la escala ESPA-29, Escala de Estilos de Socialización en la Adolescencia (Musitu y García, 2004), que permitió evaluar los estilos educativos percibidos y las dimensiones subyacentes a tales estilos; y, por otra parte, la escala MACI, Millon Adolescent Clinical Inventory (Millon, 1993), para la evaluación de la psicopatología y personalidad de los adolescentes. Los resultados obtenidos muestran una relación entre la baja aceptación e implicación parental y la depresión adolescente y entre el estilo autoritario y las alteraciones externalizantes.
\end{abstract}

Palabras clave. Estilos parentales percibidos, MACI, psicopatología, adolescentes.

\section{Perceived parental styles, psychopathology and personality in adolescence}

\begin{abstract}
The aim of this study is to examine the relationship between perceived parental styles and psychopathology and personality, as defined by Millon's model, in a clinical sample of adolescents. One hundred and twenty three outpatients from 12 to 20 years participated. Two different scales were used to obtain the data of this study. On the one hand, the ESPA-29 Scale (Musitu y García, 2004) served to assess parental styles and their underlying domensions. On the other hand, the MACI Scale (Millon Adolescent Clinical Inventory, Millon, 1993) in order to assess the teenagers' psychopathology and personality. The results obtained show an association between parental low acceptance/involvement and depression, and between authoritarian style and externalizing disorders.
\end{abstract}

Keywords: Perceived parental styles, MACI, psychopathology, adolescents

\section{INTRODUCCIÓN}

El concepto de estilos parentales ha sido ampliamente utilizado en la investigación sobre el desarrollo infanto-juvenil a lo largo de las últimas décadas. Se han elaborado diversas definiciones desde distintas perspectivas, aunque una de las más aceptadas es la proporcio-

Recibido: 11-enero-2008; aceptado: 10-octubre-2008.

Correspondencia: Borja Iglesias, Programa de Atención a la Salud Mental Infanto-Juvenil, Servicio Canario de Salud, c/ Los Martínez Escobar, n 10, $3^{\circ}$ L, 35007 Las Palmas de Gran Canaria.

Correo-e: borja.iglesias@hotmail.es nada, en su modelo integrativo, por Darling y Steinberg (1993), quienes definen estilo parental como «una constelación de actitudes hacia el niño que le son comunicadas y que, tomadas conjuntamente, crean un clima emocional en el cual las conductas de los padres son expresadas. Estas conductas incluyen tanto las dirigidas a objetivos específicos a través de las cuales los padres ejecutan sus obligaciones parentales como las conductas parentales no dirigidas a objetivos concretos, tales como gestos, expresiones faciales, cambios en el tono de voz, o expresiones espontáneas de una emoción», (p. 488). 
Particularmente influyente en el estudio de los estilos parentales ha sido la aportación de Baumrind (1971), quién delineó diferentes estilos parentales que hoy son ampliamente conocidos en la literatura científica. De acuerdo con Baumrind, los padres democráticos explican a sus hijos las razones del establecimiento de las normas, reconocen y respetan su individualidad, les animan a negociar mediante intercambios verbales, toman decisiones conjuntamente y promueven una comunicación abierta con ellos. Estos padres controlan y restringen el comportamiento de sus hijos con normas y límites claros que mantienen de modo coherente y exigen su cumplimiento. Los padres autoritarios, por su parte, se caracterizan por mantener un control restrictivo y severo sobre las conductas de sus hijos, con frecuente empleo de castigos físicos, amenazas verbales y físicas y continuas prohibiciones. Intentan moldear, controlar y evaluar la conducta y actitudes de sus hijos siguiendo unos criterios rígidos y absolutos. No fomentan la independencia e individualidad. En el estilo permisivo, por el contrario, se evita hacer uso del control, se utilizan pocos castigos, se realizan pocas demandas al niño, y se le permite regular sus propias actividades. Además, los padres permisivos se muestran tolerantes y tienden a aceptar positivamente los impulsos del niño. Posteriormente, Baumrind (1996) define los estilos parentales en función de la combinación de dos factores generales que denomina «exigencia»y «sensibilidad-receptividad». El estilo democrático se define por puntuaciones elevadas en ambos factores; el indulgente por baja exigencia y alta sensibilidad-receptividad; el autoritario por alta exigencia y baja sensibilidad-receptividad y el indulgente por bajas puntuaciones en estos dos factores.

La relación entre los estilos parentales y el funcionamiento psicológico es un tema clásico dentro del estudio del desarrollo infanto-juvenil. Diferentes estudios han constatado que el estilo democrático se asocia con índices de un funcionamiento más saludable. Baumrind (1971, 1989), por ejemplo, encontró que los hijos de padres democráticos se caracterizaban por mostrar en mayor medida conductas responsables y por estar más orientados a la independencia y al logro.
Desde los estudios de Baumrind, muchos otros investigadores han intentado definir el papel desempeñado por los estilos parentales en el desarrollo de la competencia social y la psicopatología en niños y jóvenes. Así, por ejemplo, Hasebe, Nucci y Nucci (2004) relacionan el excesivo control parental con síntomas internalizantes, cuando el control se ejerce sobre aspectos del ámbito personal de los adolescentes, no tanto cuando se ejerce sobre cuestiones convencionales. Otros trabajos, más que centrarse en indicadores globales de salud/patología, han analizado la relación entre los estilos parentales e índices específicos de psicopatología y personalidad, como la ansiedad, la depresión o el consumo de drogas y la conducta antisocial. Por ejemplo, Muris, Meesters, Morren y Moorman (2004) realizaron una investigación con una muestra no clínica de 441 adolescentes en la que encontraron que bajos niveles de calidez emocional y altos niveles de rechazo, control e inconsistencia por parte de los padres correlacionaron con altos niveles de ira y hostilidad entre los adolescentes. Enns, Cox y Larsen (2000) realizaron una investigación en la que analizaron la relación entre estilos parentales y la depresión en adultos. Se encontró una asociación significativa entre la depresión y la sobreprotección paterna, en el caso de los varones, y la ausencia de cuidado materno, en el caso de las mujeres. Martín, Bergen, Roeger y Allison (2004) realizaron un estudio, con una muestra no clínica de 2596 adolescentes, en el que encontraron que, independientemente de las puntuaciones en la dimensión de «cuidado» encontradas en ambos padres, la «intrusividad» de la madre (un componente del factor de Sobreprotección) fue el índice que se relacionó con más fuerza con la depresión mostrada por los adolescentes.

En lo que respecta al vínculo entre los estilos parentales y la vulnerabilidad al suicidio adolescente, Martín y Waite (1994), utilizando el Parental Bonding Instrument (PBI; Parker et al., 1979), estudiaron la relación entre los estilos parentales y los pensamientos, actos suicidas, y depresión en una muestra constituida por 100 adolescentes normales. Encontraron diferencias significativas en las subescalas del PBI entre 
sujetos deprimidos, no deprimidos y aquellos que mostraban pensamientos suicidas y conductas auto-lesivas. Los adolescentes que percibían a sus padres dentro del estilo «control sin afecto» (bajo cuidado y alta sobreprotección) mostraban el doble de riesgo de presentar pensamientos suicidas y conductas autolíticas.

Por lo que respecta a la conexión entre estilos parentales y trastornos del estado de ánimo, Heider, Matschinger, Bernet, Alonso y Angermeyer (2006) analizaron la relación entre los estilos parentales y los trastornos de ansiedad y depresivos en seis estados europeos, encontrando una vinculación homogénea en los diferentes países. Los principales resultados que encontraron fueron los siguientes: el bajo cuidado paternal y maternal obtuvo la asociación más significativa con la presencia de trastornos emocionales; se encontró una correlación positiva entre sobreprotección materna y alteraciones emocionales, no hallándose tal asociación en el caso de la sobreprotección paterna y no se encontró ninguna asociación significativa con el autoritarismo.

En lo referente a la relación entre los estilos parentales y los trastornos mentales presentes en población clínica, Alonso et al. (2004) 1levaron a cabo un estudio en el que analizaron la relación entre los estilos parentales percibidos y el trastorno obsesivo c ompulsivo (TOC). La muestra utilizada consistió en un primer grupo de 40 pacientes ambulatorios con diagnóstico de TOC y un segundo de 40 controles normales. Los resultados mostraron que, comparados con los controles normales, los pacientes con TOC percibían niveles más altos de rechazo por parte de sus padres. No se encontraron diferencias entre ambos grupos en la dimensión de sobreprotección. En cuanto a las dimensiones del TOC, el único subtipo que pudo predecirse en base a los estilos parentales fue el de «acumulación», que se relacionó con baja calidez emocional parental.

Otros estudios se centran en la relación entre estilos parentales y trastornos de la alimentación; es el caso de Russell, Kopec-Schrader, Rey y Beaumont (1992). Estos autores administraron el PBI (Parker et al., 1979) a un grupo de 54 pacientes adolescentes con diagnóstico de anorexia nerviosa. Las puntuaciones obte- nidas por este grupo se compararon con las de dos grupos, uno de adolescentes normales y otro de pacientes adolescentes sin diagnóstico de anorexia que habían sido derivadas a una unidad psiquiátrica para población adolescente. En líneas generales, las puntuaciones de las pacientes anoréxicas estaban más próximas al grupo de adolescentes normales que al grupo de las otras pacientes psiquiátricas. Los padres y las madres de las pacientes anoréxicas obtuvieron mayores puntuaciones en el factor de cuidado y menores en el de sobreprotección, cuando la comparación se efectuaba con los padres de otras pacientes psiquiátricas.

Otro gran ámbito de estudio en torno a los estilos parentales tiene que ver con el consumo de drogas y la conducta antisocial. Pons y Berjano (1997) encontraron que los factores con mayor poder de discriminación entre los distintos niveles de consumo alcohólico por parte de los adolescentes son las conductas parentales de reprobación, castigo y rechazo. Villar, Luengo, Gómez y Romero (2003) encontraron que los adolescentes que perciben a sus padres como permisivos tienen una menor actitud negativa ante las drogas y llevan a cabo un mayor consumo de tabaco, alcohol y cannabis. En relación a la conducta antisocial, encontraron una correlación positiva con el estilo permisivo y negativa con el democrático.

Otro ámbito importante en el estudio de los estilos parentales ha sido su asociación con la autoestima. Por ejemplo Horton, Bleau y Drwecki (2006) llevaron a cabo dos investigaciones en las que analizaban la vinculación entre los estilos parentales (calidez, supervisión y control psicológico) y el narcisismo. En ambos estudios, adicionalmente, se analizaron los datos sustrayendo de las puntuaciones de narcisismo la varianza asociada al rasgo de autoestima. El «narcisismo saludable», como los propios autores lo denominan, sería aquel que incluye el rasgo de autoestima, mientras que el «narcisismo no saludable» sería aquel al que se le ha sustraído la varianza asociada con la autoestima. Los datos mostraron que la calidez parental se asoció positivamente y la supervisión negativamente con ambos tipos de narcisismo, el saludable y el no saludable. Teniendo en cuanta que la combinación de alta calidez y 
baja supervisión sería asimilable al estilo permisivo, podríamos concluir que estos autores encontraron una vinculación entre el estilo permisivo y el narcisismo. Por otra parte, el control psicológico correlacionó positivamente con las puntuaciones de narcisismo no saludable.

Por su parte, Martínez y García (2007) llevaron a cabo un estudio en nuestro país, con una muestra no clínica, en el que analizaron el impacto de los estilos parentales en la autoestima de los adolescentes. Estos autores encontraron que los hijos de padres indulgentes obtuvieron las mayores puntuaciones en autoestima, mientras que los hijos de padres autoritarios registraron puntuaciones más bajas.

En las páginas precedentes hemos realizado una revisión de la investigación previa. En lo referente a la clasificación de los estilos parentales la mayor parte de la investigación se ha desarrollado siguiendo el modelo de Baumrind (1996). Como se habrá podido comprobar, existe una notoria ausencia de estudios realizados desde los planteamientos teóricos y metodológicos de Millon en lo referente a la psicopatología y personalidad infanto-juvenil. En nuestra opinión, la complejidad de la teoría de Millon y la relevancia que ésta tiene en sus instrumentos de evaluación constituyen un factor que puede haber contribuido a este hecho. En el presente estudio se analiza la relación entre los estilos parentales, siguiendo la clasificación de Baumrind, y la psicopatología y la personalidad en la adolescencia, siguiendo la teoría y utilizando los instrumentos de evaluación de Millon. A continuación describiremos los principales aspectos de la teoría de Millon sobre la personalidad y la psicopatología. Más adelante, describiremos el instrumento escogido en nuestra investigación para la evaluación de la personalidad y la psicopatología, el Millon Adolescent Clinical Inventory, MACI, (Millon, 1993).

Para Millon (1969) la ciencia de la personología y la psicopatología debe abarcar una sinergia entre los siguientes cuatro elementos: a) teorías; b) nosologías formales desarrolladas a partir de las teorías; c) instrumentos de evaluación empíricamente construidos, desarrollados a partir de la nosología y d) áreas diana para las intervenciones, especificadas a partir de los instrumentos de evaluación.
En 1969 Millon describe su modelo biosocial basándolo en el aprendizaje biosocial. Según este modelo, los individuos, en su vida cotidiana, aprenden a utilizar estilos de personalidad cuyas características básicas dependen de: a) su constitución biofísica, que facilita un determinado comportamiento instrumental y b) de las experiencias vividas en la que los otros actúan como fuente de refuerzo. Existen estilos vitales problemáticos que pueden hacerse patológicos y que pueden codificarse en términos de qué tipo de refuerzos busca el individuo, hacia donde se orienta para encontrarlos y cómo se las arregla para obtenerlos; todo ello constituye lo fundamental de los estilos y trastornos de personalidad que orientan las conductas de afrontamiento de cada persona.

En 1990 Millon introduce una modificación conceptual en el modelo de la personalidad y sus trastornos incorporando toda la amplitud de las teorías evolutivas, tanto en su dimensión filogenética como ontogenética. En estos términos, la personalidad puede ser considerada como la representación del estilo de adaptación, más o menos diferenciado, con que un individuo se comporta y relaciona dentro de un entorno que es característico para la especie concreta a la que pertenece. Por tanto, los trastornos de la personalidad podrían entenderse como representaciones concretas de funcionamiento individual inadaptado, que reproduce el rastro de las deficiencias, desequilibrios o conflictos que la propia especie ha mostrado en cuanto a capacidad para relacionarse con el entorno en el que se desenvuelve.

En lo relativo a la controversia clásica entre la tradición nomotética e ideográfica, Millon (1998) critica las formas extremas de ambos enfoques y defiende una perspectiva integradora que busque una síntesis entre estas dos grandes tradiciones. Para él la perspectiva nomotética pierde de vista el principal objetivo, que es el entendimiento del individuo. El enfoque ideográfico concibe al individuo como el resultado de una historia transaccional única entre factores contextuales y constitucionales, que nunca ha existido antes ni volverá a existir en el futuro. Millon reconoce el carácter único y relevante de cada biografía, pero argumenta que, por sí sola, pierde la capacidad de genera- 
lización que esperamos de la ciencia, dado que los individuos no pueden ser tan individuales que no puedan ser estudiados científicamente. Según este autor, los constructos no podrían existir sin individuos con los que puedan ser comparados ni los individuos pueden ser entendidos si no es a través de los constructos.

Respecto a otra controversia clásica, la existente entre los modelos categoriales y dimensionales, Millon (1998) no opta por ninguna de las dos y se decanta por los prototipos, como unidad de análisis de la personalidad, encuadrándose dentro de esos modelos híbridos. Para este autor los prototipos son una síntesis entre categorías y dimensiones. El constructo de prototipo reconoce la heterogeneidad de los pacientes que sufren trastornos de personalidad. Los casos prototípicos puros serían extremadamente raros, cumpliendo la mayoría de los pacientes criterios para múltiples trastornos y pudiendo presentar características subclínicas de otros estilos de personalidad.

Otra de las cuestiones centrales en el ámbito de la psicopatología, la de la continuidad entre la normalidad y la salud mental, Millon la resuelve a través de las polaridades. La noción más básica al respecto es el principio de que los rasgos de personalidad individuales se distribuyen a través de un continuo que engloba los estilos y rasgos normales, niveles intermedios de alteración y la anormalidad que implican los trastornos de personalidad. Como vimos anteriormente, para Millon (1981) desde un punto de vista teórico de aprendizaje biosocial, la personalidad se compone de categorías o pautas de afrontamiento al medio aprendidas, que son formas complejas y estables de manejarse en el entorno y que conllevan conductas instrumentales mediante las cuales los individuos logran refuerzos y evitan castigos. En relación con los modelos de clasificación, este autor plantea una continuidad entre la anormalidad y la normalidad en el funcionamiento de la personalidad. En este sentido, Millon (1991) propone que tanto los estilos de personalidad normales como los anormales se pueden derivar mediante la combinación de tres polaridades: dolor-placer, actividad-pasividad y sí mismo-otros. La polaridad «actividad-pasividad» se refiere a la capacidad de tomar la iniciativa para configurar los acontecimientos que rodean al individuo. Los sujetos activos son emprendedores para controlar las circunstancias del medio, mientras que los pasivos se caracterizan por inactividad, falta de ambición, resignación o esperar a que los acontecimientos sigan su curso. La polaridad «placer-dolor» refleja la tendencia a buscar aquellas situaciones que suponen reforzamiento positivo, en el extremo del placer, y la tendencia a emitir conductas de evitación y escape ante situaciones potencialmente dañinas, en el polo del dolor. Por último, la polaridad «sí mismo-otros» se relaciona con la dimensión introversión-extroversión y alude a la fuente de refuerzo del individuo. Los individuos dependientes sienten una elevada necesidad de apoyo $\mathrm{y}$ atención, mientras que los independientes una gran confianza en sí mismos y los ambivalentes un conflicto por depender de sí mismos o de los demás.

En cuanto a la evaluación de los trastornos de personalidad, en población adulta, Millon desarrolló el Millon Clinical Multiaxial Inventory (MCMI), del que hay tres versiones. En lo referente a la evaluación de la población adolescente, el primer instrumento que desarrolló Millon fue el Millon Adolescent Personality Inventory (MAPI), distribuido inicialmente en 1974. Posteriormente, en 1993, Millon desarro1ló el Millon Adolescent Clinical Inventory (MACI), instrumento análogo para población adolescente. Realizaremos una descripción pormenorizada de este cuestionario más adelante en este trabajo. Entre otras modificaciones en relación a su predecesor, el MACI incluyó varias escalas para la evaluación de diferentes síndromes clínicos y aumentó el número de prototipos de personalidad

Por último, nos gustaría realizar algunas consideraciones en relación a los prototipos de personalidad en la adolescencia, desde la teoría de Millon. Siguiendo el DSM-IV-TR se puede diagnosticar un trastorno de personalidad antes de los 18 años en todos los trastornos, a excepción del antisocial. Para ello tienen que cumplirse una serie de condiciones como que las características deben haber estado presentes durante al menos un año y no pueden limitarse a una etapa particular del desarrollo o a un episodio de trastornos del Eje I. Millon elude el 
término trastorno de personalidad y opta por el de prototipo de personalidad. Elegir el término prototipo en vez del de trastorno, denota su preferencia por un modelo dimensional en vez de categorial (McCann, 1999). Pero también refleja la cautela del autor, dado que la personalidad no adquiere una estabilidad y consistencia importante hasta la adultez, es arriesgado realizar estos diagnósticos a edades tempranas, sobre todo teniendo en cuenta la estigmatización, yatrogenia y efectos legales que esto puede tener. No olvidemos que para este autor los trastornos de personalidad son sistemas dinámicos, no entidades estáticas y permanentes. Por este motivo no debe sorprendernos que eluda el término trastorno en un período evolutivo que se caracteriza más por el cambio que por la estabilidad. Esta cautela se refleja también en que los nombres de los prototipos no reflejan las categorías diagnósticas del DSM, como ocurre en la versión adulta del cuestionario de Millon, el MCMI-III. A pesar de esto, cada una de estas escalas de los prototipos se relaciona específicamente con cada uno de los trastornos de personalidad del DSM.

En resumen, si hacemos un balance global de la literatura previa que analiza la vinculación entre estilos parentales y psicopatología y personalidad en la adolescencia, podemos concluir que se constata que el estilo democrático es el que se relaciona con mayor fuerza con diferentes medidas de funcionamiento adaptado a nivel psicológico y social. También tiende a encontrarse una asociación clara entre los trastornos internalizantes mostrados por los adolescentes, como la ansiedad y la depresión, y el estilo autoritario. Se constata que el estilo parental permisivo o indulgente se relaciona con más fuerza con síntomas externalizantes. Recordemos que, según la clasificación de Achenbach (1991), ya clásica en psicopatología infantojuvenil, los trastornos internalizantes incluyen el aislamiento, las quejas somáticas, la ansiedad y la depresión, mientras que los externalizantes engloban las conductas delictivas y agresivas.

El objetivo del presente trabajo es analizar la relación entre los estilos parentales y la psicopatología y la personalidad en población clínica adolescente. En este ámbito de estudio existe una notoria escasez de estudios desde los planteamientos teóricos y de evaluación de Millon, siendo estos de capital relevancia en la psicología clínica actual. El presente trabajo pretende contribuir a cubrir este llamativo vacío. Por otra parte, la mayor parte de los estudios arriba citados se han realizado con población angloparlante y muestras no clínicas, carencia que pretende cubrir el presente trabajo, al centrarse en una muestra clínica de población española. El conocer qué estilos parentales y componentes de los mismos se relacionan con alteraciones psicopatológicas y de personalidad en la adolescencia permitirá desarrollar estrategias eficaces de prevención y tratamiento.

\section{MÉTODO}

\section{Participantes}

Seleccionamos una muestra de 123 pacientes, de ambos sexos y edades comprendidas entre los 12 años y 0 meses y los 19 años y 11 meses. El 17\% de estos pacientes se obtuvo a través del Centro de Psiquiatría y Psicología de la Infancia y de la Adolescencia, clínica privada ubicada en Santiago de Compostela. E1 83\% restante se consiguió a través de la sección de salud mental infanto-juvenil de la Unidad de Salud Mental de Vecindario, situada en Las Palmas de Gran Canaria. La selección de la muestra tuvo en cuenta la edad como único criterio de inclusión, de forma que participaron en el estudio todos los pacientes, dentro del ámbito de edad objeto de estudio, que acudieron a ambos dispositivos, especializados en la atención a la salud mental infanto-juvenil. El único criterio de exclusión fue que los pacientes tuvieran un diagnóstico de retraso mental, dado que este trastorno dificulta o imposibilita la compresión de los autoinformes. En cuanto a la distribución por edades, el tanto por ciento que representa cada edad en la muestra es la siguiente: 12 años (16\%), 13 años (19\%), 14 años (18\%), 15 años (19\%), 16 años (15\%), 17 años (9\%), 18 años (2\%) y 19 años (3\%). Respecto al género, de los 123 pacientes, el 48\% eran chicas y el $52 \%$ chicos. En lo referente al nivel educativo, la distribución del tanto por ciento de cada grupo de edad en los diferentes niveles 
educativos es la siguiente: $6^{\circ}$ de primaria $(11 \%) ; 1^{\circ} \mathrm{ESO}(26 \%) ; 2^{\circ} \mathrm{ESO}(24 \%) ; 3^{\circ} \mathrm{ESO}$ $(12 \%) ; 4^{\circ}$ ESO $(18 \%) ; 1^{\circ}$ Bach $(2 \%) ; 2^{\circ}$ Bach $(3 \%) ; 1^{\circ}$ carrera $(2 \%)$ y $2^{\circ}$ carrera $(1 \%)$.

\section{Instrumentos de evaluación}

Millon Adolescents Clinical Inventory (MACI; Millon, 1993). Para la evaluación de la personalidad y la psicopatología en la adolescencia se ha utilizado el cuestionario MACI, en la adaptación española llevada a cabo por TEA. Las escalas del MACI están agrupadas en tres grupos fundamentales: prototipos de personalidad, preocupaciones expresadas y síndromes clínicos. Las 12 escalas que configuran los prototipos de personalidad del MACI son las siguientes: introvertido, inhibido, pesimista, sumiso, histriónico, egocéntrico, rebelde, rudo, conformista, oposicionista, autopunitivo y tendencia límite. Las preocupaciones expresadas se centran en sentimientos y actitudes acerca de cuestiones que tienden a preocupar a la mayoría de adolescentes con problemas; y engloban las siguientes 8 escalas: difusión de la identidad, desvalorización de sí mismo, desagrado por el propio cuerpo, incomodidad respecto al sexo, inseguridad con los iguales, insensibilidad social, discordancia familiar y abusos en la infancia. Estas escalas se centran en los sentimientos y actitudes acerca de cuestiones que tienden a preocupar a la mayoría de adolescentes con problemas. Las 7 escalas que configuran la sección de síndromes clínicos son las siguientes: trastornos de la alimentación, abuso de sustancias, predisposición a la delincuencia, propensión a la impulsividad, ansiedad, afecto depresivo y tendencia al suicidio. En cuanto a las cualidades psicométricas del MACI, los coeficientes de fiabilidad referidos a la consistencia interna (alpha) cubren un rango, en las diferentes escalas, que va desde 0,73 hasta 0,91 . La mediana del coeficiente de fiabilidad test-retest es de 0,92. En lo referente a la validez, la correlación entre las puntuaciones de las escalas y el criterio clínico es muy elevada, ya que 20 de los 24 coeficientes calculados son estadísticamente significati$\operatorname{vos}(p<0,05)$.
Escala de Estilos de Socialización Parental en la Adolescencia (ESPA-29; Musitu y García, 2004). Para la evaluación de los estilos parentales empleamos la presente escala, un instrumento que ha sido utilizado en múltiples estudios en nuestro país, que han apoyado su fiabilidad y validez como medida de estilos parentales (Marchetti, 1997; Llinares, 1998; León, 2000). Este instrumento se fundamenta en los dos ejes de socialización referidos en la literatura previa: a) «implicación-aceptación» (expresión de reacciones de aprobación y afecto cuando los hijos se comportan de acuerdo con las normas familiares) y b) «coerción-imposición» (estilo de socialización que se utiliza cuando los hijos se comportan de manera discrepante con las normas de funcionamiento familiar). Estas dos dimensiones se consideran como independientes, y de su combinación obtendríamos los siguientes cuatro estilos parentales: a) estilo autorizativo (alta aceptaciónimplicación y alta coerción-imposición); b) estilo indulgente (alta aceptación-implicación y baja coerción-imposición); c) estilo autoritario (baja aceptación-implicación y alta coerción-imposición); d) estilo negligente (puntuaciones bajas en ambas dimensiones). El estilo autorizativo seria equiparable al denominado por Baumrind y otros autores como democrático. Asimismo, se pueden obtener valoraciones pormenorizadas de los estilos de socialización de los padres en las subescalas que contribuyen a estas dos dimensiones principales: afecto, indiferencia, diálogo y displicencia en la dimensión de aceptación-implicación; coerción verbal, coerción física y privación en la dimensión de coerción-imposición. En este instrumento, un hijo valora la actuación de su padre y de su madre en 29 situaciones, obteniendo una medida para cada padre en las dimensiones aceptación-implicación y coerción-imposición. A partir de estas puntuaciones en las dos dimensiones se tipifica el estilo de socialización de cada padre como autorizativo, indulgente, autoritario o negligente. De esta forma, se asigna un padre al estilo autorizativo si tiene una puntuación superior al percentil 50 tanto en la dimensión de aceptación-implicación como en la de coerción-imposición; se asigna al estilo indulgente si tiene un percentil mayor de 50 en 
aceptación-implicación y menor en coerciónimposición; al estilo autoritario si obtiene un percentil menor a 50 en aceptación-implicación y mayor en coerción-imposición) y se clasifica como estilo negligente si alcanza percentiles menores a 50 en ambas dimensiones. En cuanto a las cualidades psicométricas del ESPA-29, se ha obtenido una elevada consistencia interna en las 7 escalas de socialización del padre y de la madre de forma independiente. El mayor coeficiente de consistencia correspondió a la escala de «afecto» de la madre (0.94), y el menor, a la «displicencia» del padre en situaciones problemáticas (0.82). Musitu y García (2004) también han llevado a cabo un análisis factorial que ha contrastado empíricamente la validez teórica del modelo bidimensional de los estilos de socialización.

\section{RESULTADOS}

Con el objetivo de analizar si existen diferencias significativas entre los estilos parentales cuando atendemos a las escalas del MACI, se utilizó el análisis de varianza, con comparaciones post hoc (prueba de Scheffé). Para analizar la asociación entre los dos factores generales de los estilos parentales y las diferentes escalas del MACI, se ha utilizado el coeficiente de correlación de Pearson.

\section{Estilos parentales, personalidad y psicopatología}

En las Tablas 1 y 2 se presentan, respectivamente, los resultados de los análisis de varianza correspondientes a estilos maternos y paternos. Los porcentajes y tamaños de los grupos que representan cada estilo parental son los siguientes. Estilo parental materno: negligente $(11 \%$, $n=13)$, indulgente $(33 \%, n=40)$, autoritario $(23 \%, n=28)$ y democrático $(34 \%, n=42)$. Estilo parental paterno: negligente $(21 \%, n=$ $26)$, indulgente $(26 \%, n=32)$, autoritario $(19 \%$, $n=24)$ y democrático $(24 \%, n=30)$.

Como se recoge en las Tablas 1 y 2 , el estilo autoritario paterno se asocia con los prototipos rudo, oposicionista y autopunitivo; mientras que el materno lo hace con el rebelde. Respecto a las preocupaciones expresadas y síndromes clínicos, el estilo autoritario se vincula con la escala de discordancia familiar en los padres y con las de abusos en la infancia e inclinación al abuso de sustancias en padres y madres. Por su parte, el estilo negligente se asocia con la escala de discordancia familiar sólo en el caso de la madre. No se encuentran vinculaciones significativas en el caso del estilo negligente paterno. El estilo indulgente se asocia con el prototipo conformista y la incomodidad respecto al sexo en ambos progenitores. El estilo democrático, por último, se asocia con el prototipo histriónico (sólo en la madre de forma significativa, observándose esta tendencia en los datos en el caso del padre).

\section{Dimensiones generales de los estilos parentales, personalidad y psicopatología}

En la Tabla 3 se recogen las correlaciones entre los factores generales aceptación/ implicación y coerción/imposición, tanto de la madre como del padre, con las diferentes escalas del MACI. En el análisis y discusión de resultados nos hemos centrado en estas dos dimensiones generales, que son las más consensuadas en este ámbito de estudio.

Los principales resultados encontrados en relación al factor aceptación/ implicación han sido los siguientes: a) correlaciones negativas con los prototipos pesimista y autopunitivo (en ambos padres) y oposicionista (madre); b) correlación positiva con el prototipo conformista (en ambos padres); c) correlaciones negativas con las escalas difusión de la identidad, discordancia familiar y abusos en la infancia (en ambos padres) y desvalorización de sí mismo (madre); d) correlación positiva entre la aceptación de ambos padres y la escala de incomodidad respecto al sexo; d) correlaciones negativas con los síndromes clínicos de afecto depresivo y tendencia al suicidio (en ambos padres) e inclinación al abuso de sustancias (madre).

Los principales resultados encontrados en relación al factor coerción / imposición han sido los siguientes: a) correlación negativa con el prototipo conformista (en ambos padres); b) correlación positiva con los prototipos rebelde 
Tabla 1. Estilo parental materno y escalas del MACI

\begin{tabular}{|c|c|c|c|c|c|c|}
\hline & Negligente & Indulgente & Autoritario & Democrático & $F$ & Scheffe $e^{a}$ \\
\hline Introvertido & $30,5 \quad(7,3)$ & $27,4(11,9)$ & $27 \quad(8,9)$ & $24,5(10,7)$ & 1,10 & \\
\hline Inhibido & $32,7(15,2)$ & $28,7(17,1)$ & $26,9(12,6)$ & $22,2(12,4)$ & 2,38 & \\
\hline Pesimista & $24,3(11,9)$ & $16,5(11,2)$ & $19,8(11,2)$ & $16,3(11,2)$ & 1,84 & \\
\hline Sumiso & $51,2 \quad(8,9)$ & $51,9(15,2)$ & $49,7(11,8)$ & $48 \quad(10,7)$ & 0,88 & \\
\hline Histriónico & $29,2(16,2)$ & $30,8(12,7)$ & $34,8(12,1)$ & $\mathbf{3 8 , 1}(11,1)$ & $3,23 *$ & D \\
\hline Egocéntrico & $26 \quad(14,1)$ & $26,2(12,1)$ & $30,5(10,2)$ & $31,3(11,8)$ & 1,94 & \\
\hline Rebelde & $25,4 \quad(9,5)$ & $22 \quad(1,7)$ & $\mathbf{2 9 , 4}(1,7)$ & $29 \quad(12,2)$ & $9,00 * *$ & $\mathbf{A}>\mathbf{I} ; \mathbf{D}>\mathbf{I}$ \\
\hline Rudo & $9,6 \quad(7,2)$ & $7,6 \quad(5,1)$ & $12,2 \quad(9,2)$ & $9,7 \quad(7,6)$ & 1,77 & \\
\hline Conformista & $40,6 \quad(9,1)$ & $\mathbf{4 8 , 5} \quad(8,8)$ & $42,5(10,7)$ & $23,2(11,7)$ & $3,13 *$ & I \\
\hline Oposicionista & $28,3 \quad(9,3)$ & $21,8(10,9)$ & $28,5(10,6)$ & $23,2(12,1)$ & 2,40 & \\
\hline Autopunitivo & $30,6(17,2)$ & $24,8(17,2)$ & $31,2(10,9)$ & $23,4(15,8)$ & 1,69 & \\
\hline Límite & $18 \quad(8,4)$ & $15,2 \quad(7,8)$ & $17,3 \quad(7,1)$ & $15,5(10,1)$ & 0,59 & \\
\hline Difusión Identidad & $18 \quad(7,1)$ & $15,5 \quad(8,3)$ & $(8,2)$ & $14,8 \quad(8,8)$ & 0,95 & \\
\hline Desvalorización & $32,7(17,3)$ & $28 \quad(18,1)$ & $29,8(13,2)$ & $24 \quad(16,2)$ & 1,2 & \\
\hline Desagrado cuerpo & $14,3 \quad(9,7)$ & $12,8 \quad(9,7)$ & $10,6 \quad(7,3)$ & $9,9 \quad(9,2)$ & 1,19 & \\
\hline Incomodidad sexo & $32,1 \quad(7,2)$ & $34 \quad(7,3)$ & $28,6 \quad(7,8)$ & $31,2 \quad(5,8)$ & $3,4^{*}$ & $\mathbf{I}>\mathbf{A}$ \\
\hline Inseguridad iguales & $14,3 \quad(7,8)$ & $12,7 \quad(9,4)$ & $12,9 \quad(8,1)$ & $9,2 \quad(6,2)$ & 2,33 & \\
\hline Insensibilidad social & $21,6 \quad(8,8)$ & $19,8 \quad(7,1)$ & $24,6 \quad(9,2)$ & $23,6 \quad(8,3)$ & 2,56 & \\
\hline Discordancia familiar & $20,6 \quad(4,8)$ & $12,7 \quad(4,7)$ & $18,2 \quad(7,4)$ & $15,6 \quad(7,2)$ & $7,13 * * *$ & $\mathbf{N}>\mathbf{A} ; \mathbf{A}>\mathbf{I}$ \\
\hline Abusos & $12,3 \quad(8,3)$ & $8,6 \quad(4,9)$ & $14,2 \quad(8,3)$ & $7,9 \quad(5,2)$ & $5,6 * *$ & $\mathbf{A}>\mathbf{I} ; \mathbf{A}>\mathbf{D}$ \\
\hline Alimentación & $16,3(11,2)$ & $13,4(10,1)$ & $13,1 \quad(8,1)$ & $11,5(12,2)$ & 0,77 & \\
\hline Sustancias & $15,3 \quad(8,2)$ & $9,7 \quad(5,5)$ & $\mathbf{1 6 , 3}(11,2)$ & $13,0 \quad(9,2)$ & $4,03 * *$ & $\mathbf{A}>\mathbf{I}$ \\
\hline Delincuencia & $19,9 \quad(5,9)$ & $18,4 \quad(6,5)$ & $21,7 \quad(8,8)$ & $22,5 \quad(6,7)$ & $2,9 *$ & \\
\hline Impulsividad & $14,6 \quad(5,2)$ & $13,4 \quad(6,4)$ & $16,3 \quad(8,2)$ & $(7,1)$ & 1,93 & \\
\hline Ansiedad & $35,2 \quad(8,4)$ & $34,6 \quad(9,2)$ & $33,4 \quad(8,2)$ & $32,1 \quad(8,2)$ & 0,81 & \\
\hline Depresión & $27,6(16,1)$ & $22,5(15,1)$ & $22,3(11,2)$ & $19 \quad(13,1)$ & 1,39 & \\
\hline Suicidio & $16,4(13,2)$ & $10,6 \quad(9,2)$ & $(9,1)$ & $10,6 \quad(8,8)$ & 1,99 & \\
\hline
\end{tabular}

Nota. Para cada estilo parental se indica la media en cada escala del MACI y, entre paréntesis, la desviación típica. ${ }^{a}$ En la prueba de Scheffé, de hallar una diferencia significativa, se indica entre qué estilos se produjo, indicando con el símbolo $>$ la dirección de tal diferencia. N: Negligente, I: Indulgente, A: Autoritario y D: Democrático. ${ }^{*} p<0,05 ;{ }^{* *} p<$ 0,$01 ; * * * p<0,001$.

(madre) y rudo (padre) y c) correlación positiva con los síndromes clínicos inclinación al abuso de sustancias y propensión a la impulsividad (madre).

\section{DISCUSIÓN}

Este estudio ha permitido definir un patrón de asociaciones entre estilos/dimensiones pa- rentales y psicopatología/personalidad en adolescentes de una muestra clínica. En primer lugar, nos gustaría comentar la relación entre los diferentes estilos parentales y las diferentes escalas del MACI que se han asociado a la depresión, como son el prototipo «pesimista», las preocupaciones «difusión de la identidad»y «desvalorización de sí mismo» y los síndromes clínicos «afecto depresivo» y «tendencia al suicido» (Millon, 1993). Los datos hallados en 
Tabla 2. Estilo parental paterno y escalas del MACI

\begin{tabular}{|c|c|c|c|c|c|c|}
\hline & Negligente & Indulgente & Autoritario & Democrático & $F$ & Scheffé \\
\hline Introvertido & $26,1(12,9)$ & $27,5 \quad(3,9)$ & $28,8 \quad(10,9)$ & $24,4 \quad(9,8)$ & 0,74 & \\
\hline Inhibido & $26,6(17,6)$ & $29,2(14,7)$ & $30 \quad(17,1)$ & $22,8 \quad(8,8)$ & 1,38 & \\
\hline Pesimista & $18,9(14,3)$ & $17,3(11,5)$ & $23,4(10,6)$ & $15 \quad(10,9)$ & 2,24 & \\
\hline Sumiso & $50,5(10,3)$ & $51 \quad(16,1)$ & $46,2(12,6)$ & $51,4 \quad(8,8)$ & 1,07 & \\
\hline Histriónico & $34,2(14,1)$ & $30,2(13,2)$ & $31,1 \quad(15,9)$ & $38 \quad(8,2)$ & 2,4 & \\
\hline Egocéntrico & $29,1(14,1)$ & $25,5(10,8)$ & $27 \quad(13,1)$ & $32 \quad(11,9)$ & 1,9 & \\
\hline Rebelde & $26,4 \quad(9,8)$ & $23,3(10,2)$ & $27 \quad(14,5)$ & $23,2 \quad(7,7)$ & 2,29 & \\
\hline Rudo & $9,0 \quad(6,8)$ & $7,9 \quad(5,2)$ & $\mathbf{1 4 , 7}(11,1)$ & $8,4 \quad(4,8)$ & $3,85^{*}$ & $\mathbf{A}>\mathbf{I}$ \\
\hline Conformista & $43,6 \quad(8,8)$ & $\mathbf{4 8 , 7} \quad(9,3)$ & $37,9(11,2)$ & $46,1(10,3)$ & $5,7 * *$ & $\mathbf{I}>\mathbf{A} ; \mathbf{D}>\mathbf{A}$ \\
\hline Oposicionista & $24,1 \quad(9,8)$ & $22,5(12,4)$ & $32 \quad(10,1)$ & $21,5(10,9)$ & $4,37 * *$ & $\mathbf{A}>\mathbf{I} ; \mathbf{A}>\mathbf{D}$ \\
\hline Autopunitivo & $28,1(19,3)$ & $24,5(11,3)$ & $\mathbf{3 4 , 9}(11,3)$ & $22,6(14,9)$ & $2,94 *$ & $\mathbf{A}$ \\
\hline Límite & $16,4(10,2)$ & $15,6 \quad(6,1)$ & $18,5 \quad(6,1)$ & $14,8 \quad(9,4)$ & 0,86 & \\
\hline Difusión identidad & $16,8(10,1)$ & $14,6 \quad(7,2)$ & $20,2 \quad(7,4)$ & $14,4 \quad(8,7)$ & 2,46 & \\
\hline Desvalorización & $28,8(20,2)$ & $28 \quad(16,7)$ & $33,7(15,2)$ & $24 \quad(15,1)$ & 1,49 & \\
\hline Desagrado cuerpo & $10,5 \quad(8,9)$ & $13,3 \quad(9,9)$ & $13,6 \quad(7,7)$ & $10 \quad(9,8)$ & 1,19 & \\
\hline Incomodidad sexo & $31,3 \quad(8,1)$ & $\mathbf{3 3 , 7} \quad(6,2)$ & $(8,2)$ & $33,3 \quad(3,8)$ & $5,65^{* *}$ & $\mathbf{I}>\mathbf{A} ; \mathbf{D}>\mathbf{A}$ \\
\hline Inseguridad iguales & $2,1 \quad(9,1)$ & $13,2 \quad(7,7)$ & $(8,8)$ & $9,3 \quad(3,3)$ & 1,9 & \\
\hline Insensibilidad social & $21,7 \quad(9,2)$ & $20,9 \quad(5,8)$ & $24,7(11,7)$ & $21,9 \quad(5,8)$ & 1,05 & \\
\hline Discordancia familiar & $17,0 \quad(6,1)$ & $12,9 \quad(5,2)$ & $\mathbf{2 0 , 3} \quad(6,2)$ & $13,9 \quad(5,8)$ & $7,96 * * *$ & A $>$ I $; \mathbf{A}>\mathbf{D}$ \\
\hline Abusos & $10,7 \quad(8,5)$ & $9,7 \quad(5,8)$ & $(7,1)$ & $6,7 \quad(7,8)$ & $6,16 * *$ & $\mathbf{A}>\mathbf{D}$ \\
\hline Alimentación & $12,6(11,1)$ & $13,5(11,2)$ & $15,2 \quad(9,1)$ & $13,2(12,1)$ & 0,57 & \\
\hline Sustancias & $12,8 \quad(8,3)$ & $10,7 \quad(6,8)$ & $\mathbf{1 8 , 7}(10,5)$ & $10,9 \quad(5,8)$ & $5,15^{* *}$ & $\mathbf{A}>\mathbf{I} ; \mathbf{A}>\mathbf{D}$ \\
\hline Delincuencia & $21,0 \quad(7,6)$ & $18,5 \quad(5,7)$ & $21,9(10,8)$ & $20,8 \quad(6,2)$ & 1,26 & \\
\hline Impulsividad & $15,3 \quad(8,2)$ & $14 \quad(7,7)$ & $16,1 \quad(7,7)$ & $15,7 \quad(6,1)$ & 0,51 & \\
\hline Ansiedad & $35,1 \quad(8,8)$ & $34,4 \quad(8,8)$ & $32,3 \quad(9,9)$ & $33,5 \quad(6,9)$ & 0,50 & \\
\hline Depresión & $22,8(16,2)$ & $22,4(12,9)$ & $25,6(14,8)$ & $18,9(12,1)$ & 1,07 & \\
\hline Suicidio & $13,4(11,1)$ & $11,5 \quad(0,17)$ & $17,2(10,5)$ & $9,5 \quad(8,9)$ & 2,50 & \\
\hline
\end{tabular}

Nota. Para cada estilo parental se indica la media en cada escala del MACI y, entre paréntesis, la desviación típica. ${ }^{a}$ En la prueba de Scheffé, de hallar una diferencia significativa, se indica entre qué estilos se produjo, indicando con el símbolo $>$ la dirección de tal diferencia. N: Negligente, I: Indulgente, A: Autoritario y D: Democrático. ${ }^{*} p<0,05$; $* * p<0,01 ; * * * p<0,001$.

la presente investigación indican que el estilo negligente materno y el autoritario paterno son los que tienden a obtener las mayores puntuaciones en estas escalas relacionadas con la depresión, aunque estas diferencias no fueron significativas. Por otra parte, la investigación previa señala una mayor asociación entre la depresión y el estilo autoritario (Parker et al., 1979; Martin y Waite, 1994; Martin et al., 2004; Heider et al., 2006). Estos datos podrían expli- carse en base a lo que tienen en común los estilos negligente y autoritario, ya que ambos comparten puntuaciones bajas en el factor aceptación/implicación. De hecho, nuestros datos indican una asociación significativa entre puntuaciones bajas en el factor aceptación/implicación y todas estas escalas relacionadas con la depresión en ambos padres. Estos datos son coherentes con las investigaciones previas en las que se asociaba el bajo cuidado con la de- 
Tabla 3. Correlaciones entre la aceptación/implicación y coerción/imposición, del padre y de la madre, y las escalas del MACI

\begin{tabular}{|c|c|c|c|c|}
\hline & \multicolumn{2}{|c|}{ Aceptación / implicación } & \multicolumn{2}{|c|}{ Coerción / imposición } \\
\hline & madre & padre & madre & padre \\
\hline Introvertido & $-0,14$ & $-0,08$ & $-0,04$ & 0,08 \\
\hline Inhibido & $-0,16$ & $-0,12$ & $-0,06$ & 0,07 \\
\hline Pesimista & $-0,22 *$ & $-0,23 *$ & $-0,06$ & 0,09 \\
\hline Sumiso & $-0,00$ & 0,00 & $-0,02$ & $-0,01$ \\
\hline Histriónico & 0,08 & 0,13 & $-0,15$ & $-0,01$ \\
\hline Egocéntrico & 0,05 & 0,07 & 0,08 & $-0,00$ \\
\hline Rebelde & $-0,11$ & $-0,16$ & $0,24 * *$ & 0,12 \\
\hline Rudo & $-0,17$ & $-0,11$ & 0,17 & $0,19 *$ \\
\hline Conformista & $0,21 *$ & $0,19 *$ & $-0,20 *$ & $-0,20 *$ \\
\hline Oposicionista & $-0,23 *$ & $-0,17$ & 0,14 & 0,16 \\
\hline Autopunitivo & $-0,27 * *$ & $-0,22 *$ & 0,09 & 0,14 \\
\hline Límite & $-0,17$ & $-0,14$ & 0,06 & 0,08 \\
\hline Difusión de la identidad & $-0,23 * *$ & $-0,19 *$ & 0,03 & 0,14 \\
\hline Desvalorización sí mismo & $-0,21 *$ & $-0,15$ & 0,04 & 0,12 \\
\hline Desagrado propio cuerpo & $-0,09$ & $-0,00$ & $-0,01$ & 0,06 \\
\hline Incomodidad sexo & $0,20 *$ & $0,36 * *$ & $-0,05$ & $-0,18$ \\
\hline Inseguridad iguales & $-0,14$ & $-0,14$ & $-0,07$ & 0,07 \\
\hline Insensibilidad social & $-0,07$ & $-0,01$ & 0,12 & 0,05 \\
\hline Discordancia familiar & $-0,36 * *$ & $-0,34 * *$ & 0,14 & 0,08 \\
\hline Abusos & $-0,32 * *$ & $-0,25 * *$ & 0,13 & 0,09 \\
\hline Alimentación & $-0,11$ & $-0,02$ & $-0,02$ & 0,09 \\
\hline Sustancias & $-0,25 * *$ & $-0,17$ & $0,18^{*}$ & 0,17 \\
\hline Delincuencia & $-0,01$ & $-0,02$ & 0,15 & 0,04 \\
\hline Impulsividad & $-0,07$ & $-0,06$ & $0,18 *$ & 0,05 \\
\hline Ansiedad & $-0,08$ & $-0,09$ & $-0,07$ & $-0,07$ \\
\hline Depresión & $-0,18 *$ & $-0,18 *$ & $-0,02$ & 0,08 \\
\hline Suicidio & $-0,22 *$ & $-0,21 *$ & 0,07 & 0,10 \\
\hline
\end{tabular}

$* p<0,05 ; * * p<0,01 ; * * * p<0,001$.

presión (Enns et al., 2000; Martín et al., 2004; Heider et al., 2006) y el suicidio (Martín y Waite, 1994).

En relación al prototipo conformista, se encuentra una asociación entre este prototipo de personalidad y el estilo indulgente $y$, consecuentemente, una correlación positiva con el factor aceptación/implicación y negativa con el factor coerción/imposición, siempre en ambos progenitores. No hemos encontrado una explica- ción satisfactoria de este resultado basándonos en las teorías y datos previos. Por una parte, Millon (1993) señala que el prototipo de personalidad conformista es semejante al trastorno obsesivo compulsivo de la personalidad del DSM y que son sujetos que han estado sujetos a obligaciones y disciplina y que parecen haber sido obligados a aceptar los valores que otros les han impuesto. Teniendo en cuenta la definición de este prototipo y los planteamientos 
etiológicos de Millon, deberíamos esperar una asociación entre el prototipo conformista y el estilo autoritario. Por otra parte, en el estudio de Alonso et al. (2004) veíamos que se había encontrado que los pacientes con TOC percibían niveles más altos de rechazo por parte de sus padres, encontrándose que el subtipo de «acumulación» se asociaba con baja calidez emocional parental. Este factor de rechazo podría ser considerado como el reverso del factor de aceptación-implicación del ESPA29. Consideramos que sería necesaria investigación adicional para poder dar una explicación satisfactoria a estos datos.

Nuestro estudio también permite perfilar el patrón de relaciones que se establece entre los estilos parentales y diferentes escalas del MACI que podríamos denominar externalizantes (prototipos rebelde, rudo y oposicionista; preocupación expresada de la discordancia familiar y síndromes clínicos de inclinación al abuso de sustancias, predisposición a la delincuencia y propensión a la impulsividad). Para realizar esta agrupación nos hemos basado en el estudio de Blumentritt (2004), en el que se analizan las relaciones entre diferentes escalas del MACI y entre estas y diferentes trastornos mentales, siguiendo la clasificación del DSM.

Se encuentra una asociación significativa entre el estilo autoritario paterno y los prototipos rudo y oposicionista y entre el estilo autoritario materno y el rebelde. Como vimos en la introducción, en algunas investigaciones (Muris et al., 2004) se había encontrado una asociación entre el estilo autoritario y la hostilidad de los adolescentes. Podríamos considerar que la hostilidad es un elemento presente en los prototipos rebelde, rudo y oposicionista. En relación al resto de las escalas del MACI, existe una clara asociación, en ambos padres, entre el estilo autoritario y la discordancia familiar y el síndrome de inclinación al abuso de sustancias. Estos resultados no son coincidentes con algunas investigaciones previas, como la de Villar et al. (2003), en la que se había encontrado una asociación entre el estilo indulgente y la conducta antisocial y el consumo de drogas. Una posible explicación a esta discrepancia podría ser la diferente naturaleza de las muestras objeto de estudio, tratándose la nuestra de una muestra clínica y la del estudio de Villar de una muestra comunitaria.

La relación entre el factor de aceptación/ implicación y las escalas externalizantes del MACI también es clara, ya que existe una correlación negativa con todas ellas, siendo estas correlaciones significativas en la mayor parte de los casos. Esta asociación entre baja aceptación e implicación y las alteraciones externalizantes tendría dos posibles significados. Una posible explicación podría ser que hay una relación directa entre esta carencia, a nivel afectivo, y el desarrollo de las alteraciones externalizantes. Otra posibilidad es que la coerción y la imposición, cuando no van acompañadas de diálogo y afecto tienen un efecto más pernicioso. La coerción, en este último caso sería más dura al no ir amortiguada por este elemento afectivo.

En relación al factor coerción/imposición, encontramos una correlación positiva entre esta dimensión y las escalas externalizantes. Los anteriores resultados son coherentes con algunas investigaciones previas. Como veíamos en la introducción, Pons y Berjano (1997) relacionaban el abuso de sustancias, en concreto del alcohol, con la reprobación, el castigo y el rechazo parental.

Por otra parte, también es interesante prestar atención a los resultados obtenidos con algunas escalas concretas del MACI. Por ejemplo, es interesante constatar que no se ha encontrado ninguna asociación significativa entre el prototipo egocéntrico (narcisista) y la desvalorización de si mismo con ninguno de los estilos parentales. Como vimos en la introducción, Horton et al. (2006) habían encontrado una asociación entre el estilo indulgente (alta calidez y baja supervisión) y la personalidad narcisista y la autoestima. Martínez y García (2007) encontraron una asociación entre estilo indulgente y alta autoestima. Teniendo en cuenta estos datos previos, hubiéramos esperado una asociación entre el estilo indulgente y puntuaciones altas en el prototipo egocéntrico y bajas en la escala de desvalorización de sí mismo.

Otro dato reseñable es que no se observa ninguna asociación entre los estilos parentales, ni ninguno de los diferentes componentes, y la escala de trastornos de la alimentación, ni tam- 
poco con respecto a otra escala muy relacionada como es la de desagrado con el propio cuerpo. Ciertamente, este es un dato que nos ha sorprendido, a pesar de que ya Rusell et al. (1992) encontraron que los estilos parentalales de los padres de las pacientes anoréxicas estaban más próximas al grupo de adolescentes normales que a otro grupo constituido por otras pacientes psiquiátricas.

En síntesis, y como conclusiones más destacables, nuestro estudio muestra que existe una fuerte asociación entre la baja aceptación/implicación de ambos padres y las escalas relacionadas con la depresión. Se constata, asimismo, una clara vinculación entre el prototipo conformista y el estilo indulgente, en ambos progenitores. Con respecto a las alteraciones externalizantes, existe una clara asociación con el estilo autoritario, en ambos progenitores.

Los datos aportados en la presente investigación, dada su procedencia clínica, pueden tener una importante aplicabilidad en este ámbito; conocer la asociación existente entre los estilos parentales y las diferentes alteraciones psicológicas de los adolescentes nos permitirá saber sobre que conductas parentales concretas debe incidir la prevención y el tratamiento psicológico en cada una de estas alteraciones para una adecuada superación de las mismas. La escasez de investigaciones de tipo clínico en este ámbito otorga, si cabe, un valor añadido a estos datos.

Una de las limitaciones de esta investigación es que no es posible determinar si las prácticas parentales son causa o consecuencia de las alteraciones presentadas por los hijos. Es posible que las propias características del adolescente sean la responsables del estilo parental, de forma que un hijo bien adaptado genere un estilo más democrático, mientras que un adolescente con graves trastornos de conducta provoque un mayor autoritarismo. Como han puesto de manifiesto otros estudios, utilizando datos longitudinales, las dimensiones parentales y los problemas de conducta parecen afectarse recíprocamente (Romero, Luengo y Gómez-Fraguela, 2000). Siguiendo en esta línea, la propia psicopatología del hijo también puede estar condicionando la percepción de los estilos parentales. Es posible que un hijo de- presivo perciba a sus padres como poco afectuosos, no porque realmente lo sean, sino como consecuencia de un sesgo negativo derivado de la propia depresión del hijo. Partiendo de un modelo teórico integrativo, nuestra hipótesis es que estos factores se retroalimentan mutuamente; pero esta hipótesis sólo podría ser contrastada a través de un estudio longitudinal. Otra limitación de esta investigación es que la información obtenida se deriva de los autoinformes de los adolescentes, por lo que deberíamos hablar de estilos parentales percibidos más que de estilos parentales. Desde una epistemología constructivista, cualquier evaluación realizada de los estilos parentales, por un observador externo o por autoinformes de los propios padres, estaría sesgada, dado que los estilos parentales «reales» no existen como una entidad objetiva independiente del observador o instrumento de medida utilizado. Consideramos que los autoinformes de los adolescentes constituyen el método de evaluación más objetivo para la evaluación de este constructo, en comparación con otras alternativas disponibles. Diferentes autores y estudios han analizado las discrepancias entre la percepción de los adolescentes y la de los padres sobre los estilos educativos de estos últimos; y han concluido que la correlación con un observador externo es mayor con los autoinformes de los adolescentes que con los autoinformes de los padres (Steinberg et al., 1994; Smetana, 1995). Adicionalmente, estamos de acuerdo con Wolfradt et al. (2003) en que para medidas de personalidad y psicopatología, parece más importante estudiar las percepciones de los adolescentes de los estilos educativos parentales, que buscar medidas más objetivas o estudiar la evaluación de los padres de su propia conducta. Sea la percepción del adolescente la que más se adecua a la realidad o no, esta percepción es su realidad y la que tendrá relación con su psicopatología y personalidad y la que es objeto de estudio en la presente investigación. A pesar de este hecho, reconocemos la importancia y la necesidad de investigar la relación entre los estilos parentales y el ajuste adolescente usando múltiples métodos y diferentes fuentes de información; como recomiendan Villar et al. (2003) 
En cuanto a líneas futuras de investigación, un punto en el que todavía quedan muchas preguntas sin responder es el referente a las discrepancias en los estilos parentales de ambos progenitores y su posible relación con la psicopatología y la personalidad en la adolescencia. A nivel teórico, los autores clásicos no llamaron excesivamente la atención sobre este aspecto. En cuanto a los datos disponibles actualmente en población adolescente normal, el estudio de Musitu y García (2004) encontró que los estilos parentales de ambos progenitores son percibidos por los adolescentes como altamente coincidentes, siendo las diferencias encontradas de índole más cuantitativa que cualitativo. Una pregunta que todavía está por responder es si existe una mayor discrepancia en población clínica y su posible repercusión a nivel psicopatológico.

Por último, un aspecto crucial que todavía esta sin resolver es el tipo de relación entre los estilos parentales y la psicopatología y la personalidad es lineal, curvilínea o interactiva, o en que tipo de casos se producen unos tipos de relación u otros. En cualquier caso, este estudio deja constancia de la relevancia de los estilos parentales para entender la psicopatología adolescente, también en muestras clínicas, y avala la importancia de considerar estos estilos a la hora de diseñar estrategias de intervención.

\section{REFERENCIAS}

Achenbach, T. M. (1991). Integrative guide for the 1991 CBCL/4-18, YSR and TRF profiles. Burlington, VT: University of Vermont Department of Psychiatry.

Alonso, P., Menchón, J., Mataix-Cols, D., Pifarré, J., Urretavizcaya, M., Crespo, J., Jiménez, S., Vallejo, G., y Vallejo, J. (2004). Perceived parental style in obsessive-compulsive disorder: relation to symptom dimensions. Psychiatry Research, 127, 267-78.

Baumrind, D. (1971). Current patterns of parental authority. Developmental Psychology Monograph, 4, 1-103.

Baumrind, D. (1989). Rearing competent children. En W. Damon (Ed.), New direction for child development: Child development, today and tomorrow (pp. 349-378). San Francisco: Jossey-Bass.

Baumrind, D. (1996). The discipline controversy revisited. Family Relations, 45, 405-414. Nacional Council on Family Relations.
Blumentritt, J. (2004). MACI personality patterns and DSM-IV symptoms in a simple of troubled MexicanAmerican adolescents. Journal of Child and Family Studies, 13, 163-178.

Darling, N., y Steinberg, L. (1993). Parenting style as a context: an integrative model. Psychological Bulletin, 113, 487-496.

Gonzales, N.A., y Cauce, A. M. (1996). Interobserver agreement in the assessment of parental behavior and parent-adolescent conflict: African American mothers, daughters, and independent observers. Child Development, 67, 1483-1498

Enns, M., Cox., B., y Larsen, D. (2000). Perceptions of parental bonding and symptom severity in adults with depression: mediation by personality dimensions. $\mathrm{Ca}$ nadian Journal of Psychiatry, 45, 263-268.

García-Linares, M.C., Peregrina, S., y Lendínez, J. (2002). Los estilos educativos de los padres y la competencia psicosocial de los adolescentes. Anuario de Psicología, 33, 79-85.

Hasebe, Y., Nucci, L., y Nucci, M. (2004). Parental control of the personal domain and adolescent symptoms of psychopathology: a cross-national study in the United Status and Japan. Child Development, 75, 815-828.

Heider, D., Matschinger, H., Bernet, S., Alonso, J., y Angermeyer, M. (2006). Relationship between parental bonding and mood disorder in six European countries. Psychiatry Research, 143, 89-98.

Horton, R.; Bleau, G., y Drwecki, B. (2006). Parenting narcissus: what are the links between parenting and narcissism? Journal of personality, 74, 345-76.

León, J. (2000). La contrastación de un modelo bidimensional en la socialización parental de adolescentes. Trabajo de investigación. Dir. Fernando García, Dpto. De Metodología. Facultad de Psicología. Universidad de Valencia.

Llinares, L. (1998). La configuración del autoconcepto y los valores en el contexto familiar. Tesis doctoral. Dirs.: G., Musitu y M. Á. Molpeceres. Facultad de Psicología. Universidad de Valencia

Marchetti, B. (1997). Concetto di se'relazioni familiari e valori. Tesis de Licenciatura en Psicología Social. Universita degli Studi di Bologna.

Martin, G., y Waite, S. (1994). Parental bonding and vulnerability to adolescent suicide. Acta Psychiatrica Scandinavica, 89, 246-54.

Martin, G., Bergen, H.A., Roeger, L., y Allison, S. (2004). Depression in young adolescents: Investigations using 2 and 3 factor versions of the parental bonding instrument. Journal of Nervous and Mental Disorders, 192, 650-657.

Martínez, I., y García, J.F. (2007). Impact of parenting styles on adolescents' self-esteem and internalization of values in Spain. The Spanish Journal of Psychology, 10, 338-348. 
McCann, J. (1999). Assessing adolescents with the MACI: using the Millon Adolescent Clinical Inventory.

Millon, T. (1976). Psicopatología moderna: enfoque biosocial de los aprendizajes erróneos y de los disfuncionalismos. Barcelona: Salvat (Trabajo original publicado en inglés en 1969).

Millon, T. (1990). Toward a new personology: An evolutionary model. New York: Wiley.

Millon, T. (1991). Classification in psychopathology: Rationale, Alternatives and Standard. Journal of Abnormal Psychology, 100, 245-261.

Millon, T. (1993). MACI, Millon Adolescent Clinical Inventory. Madrid: TEA.

Millon, T., y Davis, R.D. (1998). Trastornos de la personalidad. Más allá del DSM-IV. Barcelona: Masson (Trabajo original publicado en 1996).

Muris, P., Meesters, C., Morren, M., y Moorman, L. (2004). Anger and hostility in adolescents: relationship with self-reported attachment style and perceived parental rearing styles. Journal of Psychosomatic Research, 57, 257-64.

Musitu, G., y García, F. (2004). ESPA29, Escalas de estilos de socialización en la adolescencia ( $2^{\mathrm{a}} \mathrm{ed}$.). Madrid: TEA ediciones.

Parker, G., Tupling, H., y Brown, L.B. (1979). A parental bonding instrument. British Journal of Medical Psychology, 52, 1-10.
Pons, J., y Berjano, E. (1997). Análisis de los estilos parentales de socialización asociados al abuso de alcohol en adolescentes. Psicothema, 9, 609-617.

Romero, E., Luengo, M.A., y Gómer-Fraguela, J.A. (2000). Factores psicosociales y delincuencia: un estudio de efectos recíprocos. Escritos de Psicología, 4, 78-91.

Russell, K., Kopec-Schrader, E., Rey, J.M., y Beaumont, P.J. (1992). The parental bonding instrument in adolescent patients with anorexia nervosa. Acta Psychiatrica Scandinavica, 86, 236-9.

Smetana, J. G. (1995). Parenting styles and conceptions of parental authority during adolescence. Child Development, 66, 299-315.

Steinberg, L., Lamborn, S., Darling, N., Mounts, N., y Dornbusch, S. (1994). Over-time changes in adjustment and competence among adolescents from authoritative, authoritarian, indulgent, and neglectful families. Child Development; 65, 754-770.

Villar, P., Luengo, M. A., Gómez, J. A., y Romero, E. (2003). Una propuesta de evaluación de variables en la prevención de la conducta problema en la adolescencia. Psicothema, 15, 581-588.

Wolfradt, U., Hempel, S., y Miles, J. (2003). Perceived parenting styles, despersonalisation, anxiety and coping behaviours in adolescents. Personality and Individual Differences. 34, 521-532. 